\title{
Die Agonie des wundärztlichen Berufsstandes am Beispiel des Standespolitikers Arnold Schlegel (1850-1924)
}

Dominik Gross

\section{Summary}

The name of Arnold Schlegel stands for a dazzling and complex personality. After being apprenticed to a barber surgeon in Württemberg, he spent about four decades in Switzerland working as an innkeeper. Yet, Schlegel distinguished himself as one of the leading representatives of the last nonacademic surgeons in Württemberg. In contrast to most of his surgical colleagues, most of Schlegel's vocational activities are documented. This study is based on unpublished documents of different archives of Württemberg and Switzerland. The aim of the study is to illuminate the life of Arnold Schlegel and, in view of his policy, to illustrate the trying circumstances of contemporary barber surgery.

\section{Zusammenfassung}

Hinter dem Namen Arnold Schlegel verbirgt sich eine ebenso schillernde wie widersprüchliche Persönlichkeit. Nachdem er in Württemberg eine Ausbildung zum Wundarzt absolviert hatte, lebte Schlegel über einen Zeitraum von annähernd vier Jahrzehnten als Gastwirt in der Schweiz. Gleichwohl trat er als einer der führenden Interessenvertreter des aussterbenden württembergischen Wundärztestandes hervor. Anders als bei den meisten Handwerkschirurgen sind Schlegels berufliche Aktivitäten weitgehend überliefert. Die vorliegende Arbeit stützt sich hierbei auf unveröffentlichte Dokumente aus verschiedenen Archiven Württembergs und der Schweiz. Ziel des Beitrags

Dr. Dr. Dominik Gross, M.A., Institut für Geschichte der Medizin der Universität Würzburg, Oberer Neubergweg 10a, D-97074 Würzburg 
ist es, über eine Lebensbeschreibung hinaus am Beispiel des Standespolitikers Schlegel die schwierige Lage der zeitgenössischen Wundärzte anschaulich zu machen.

\section{Einleitung}

Obgleich die chirurgische Tätigkeit bis weit in das 19. Jahrhundert hinein vornehmlich in den Händen handwerklich ausgebildeter Wundärzte $\operatorname{lag}^{1}$, wurde die betreffende Berufsgruppe im Unterschied zur akademischen Ärzteschaft bisher kaum systematisch untersucht ${ }^{2}$. Das bestehende Forschungsdefizit wird unter anderem darauf zurückgeführt, dass die nichtakademischen Chirurgen «für die Nachwelt weitgehend stumm» geblieben sind ${ }^{3}$.

Eine der wenigen Ausnahmen stellt der württembergische Wundarzt Arnold Schlegel (1850-1924) dar. Seine Biographie verdient gleich in mehrfacher Hinsicht Aufmerksamkeit: So trat Schlegel nicht nur als Wund- und Hebarzt hervor, sondern auch als Standespolitiker, als Autor chirurgischer, standespolitischer sowie medizingeschichtlicher Abhandlungen, als Zeitschriftenredakteur und zudem seit 1883 als Gastwirt im traditionsreichen «Gasthof zum Bären〉 in Einsiedeln/Schweiz.

Dass die Person Arnold Schlegel dennoch bisher kaum Beachtung gefunden hat, kann mit dem ungewöhnlichen Lebenslauf erklärt werden: Mehrere Orts- und Berufswechsel hatten zur Folge, dass die betreffenden Personalakten über einzelne Archive verstreut und somit schwer zugänglich sind. Der vorliegende Beitrag stützt sich im wesentlichen auf Dokumente des Hauptstaatsarchivs Stuttgart, des Staatsarchivs Ludwigsburg, des Universitätsarchivs Tübingen, des Bezirksarchivs Einsiedeln/Schweiz, des Bürger-

1 Noch 1859 praktizierten etwa im Königreich Württemberg 796 Wundärzte, aber lediglich 442 Ärzte: vgl. Württembg Jahrb Statistik Landesk. 1859, T. I, S. 198.

$2 \mathrm{Zu}$ den wenigen Ausnahmen gehört die Promotionsarbeit von Sabine Sander zur Sozialgeschichte der württembergischen Wundärzte im 18. Jahrhundert: Dies., Handwerkschirurgen. Sozialgeschichte einer verdrängten Berufsgruppe, Göttingen 1989 (= Kritische Studien zur Geschichtswissenschaft, 83); vgl. auch dies., Heilkunde als Handwerk - Medizin in Bietigheim vor 200 Jahren. Blätter Stadtgesch 10, 1992, S. 35-56. Demgegenüber fehlt bis dato eine Gesamtdarstellung zur württembergischen Wundärzteschaft des 19. und beginnenden 20. Jahrhunderts. Für Einzelfragen vgl. die Vorstudien von C. Weiss, H. Specker, H. J.Winckelmann, Die Medizinalgesetzgebung in Württemberg im 19. Jahrhundert. Unter besonderer Berücksichtigung des Chirurgen- und Baderwesens, Münch Med Wschr 125,1983, S. 1005-1010; S. Sander, Handwerkliche Wundarznei in der Zeit der Auflösung des traditionellen Gesundheitswesens: das Beispiel Johannes Villingers (1793-1847) in Waiblingen, in: Jahrbuch des Instituts für Geschichte der Medizin der Robert-Bosch-Stiftung, Bd. 5 für das Jahr 1986, Stuttgart 1987, S. 87-128; D. Gross, Die Aufhebung des Wundarztberufs im Spiegel zeitgenössischer wundärztlicher Verlautbarungen. WMM 14, 1996 (im Druck).

3 Sander, Handwerkliche Wundarznei (wie Anm. 2), S. 87 (Zitat). 
meisteramts Dürmentingen, des Stadtarchivs Ravensburg sowie auf eine Vielzahl der zwischen 1874 und 1919 erschienenen Bände der ¿Zeitschrift für Wundärzte und Geburtshelfer> (ZWG). Einzelne von Schlegel verfasste Monographien vervollständigen den Quellenbestand.

Obgleich das Leben Schlegels - schon aufgrund der verschiedenen beruflichen Tätigkeiten - nicht als repräsentativ für die Mehrheit der 〈Handwerkschirurgen ${ }^{4}$ gelten kann, lässt doch gerade seine Tätigkeit als standespolitischer Vertreter Rückschlüsse auf die berufliche Situation der württembergischen Wundärzte zu. Ziel der vorliegenden Arbeit ist es, am Beispiel des Berufspolitikers Schlegel die besondere Lage sowie die Forderungen des aussterbenden Wundarztstandes anschaulich zu machen.

\section{Arnold Schlegel und die wundärztliche Berufsgruppe}

Arnold Schlegel wurde am 18. Mai 1850 im württembergischen Dürmentingen/Oberamt Riedlingen geboren ${ }^{5}$. Wie viele angehende Wundärzte entstammte auch Schlegel einer Handwerkerfamilie ${ }^{6}$ : Sein Vater, der 1817 geborene Fridolin Schlegel, war in Dürmentingen als Nagelschmied tätig. Seine Mutter Rosa Schlegel, geb. Probst, hatte vor dem Jahr 1850 bereits drei Söhne zur Welt gebracht, die jedoch ausnahmslos vor Vollendung ihres ersten bzw. zweiten Lebensjahres verstorben waren. Der letztgeborene Arnold wuchs daher als Einzelkind auf. Über die Jugend Schlegels liegen nur wenige Angaben vor. Aus einer späteren Bittschrift wissen wir, dass er keine höhere Schulbildung erlangte. Unterstellt man somit den typischen Lebenslauf eines angehenden württembergischen Wundarztes, so dürfte Schlegel mit 14 oder 15 Jahren eine - in der Regel dreijährige - Chirurgenlehre absolviert haben und danach als Geselle «in Condition» gegangen sein?

4 Der Begriff 〈Handwerkschirurg〉 ist aus philologischer Sicht aufgrund der implizierten Tautologie (gr. $\chi \varepsilon 1 \rho o u \rho \gamma o ́ s=$ Handarbeiter) nicht ganz unumstritten. Gleichwohl fand er Eingang in die Fachliteratur. Zur Verwendung des Begriffs siehe auch J. Oehme, Ausbildung und Bedeutung der Handwerkschirurgen unter besonderer Berücksichtigung der Ausbildung am anatomisch-chirurgischen Institut in Braunschweig, WMM 10, 1992, S. 293-301.

5 Vgl. Bürgermeisteramt Dürmentingen, Familienregister, Bd. 1, Bl. 104 sowie Universitätsarchiv Tübingen (i. f. z. als UAT) 5/39, Hospitantenbuch, 1871.

6 Zur sozialen Herkunft der Wundärzte vgl. D. Gross, Berufsvererbung und soziale Herkunft der württembergischen Wundärzte im 19. Jahrhundert, Sudhoffs Arch 80, H. 1, 1996 (im Druck).

7 Entsprechende biographische Angaben sind den wundärztlichen Prüfungsprotokollen zu entnehmen: vgl. etwa Staatsarchiv Ludwigsburg (i. f. z. als StAL) E 162 I, Bü 603, 607, 608-613 sowie UAT 14/17. In der zweiten Hälfte des 19. Jahrhunderts wurden die betreffenden Eintragungen jedoch immer summarischer. In bezug auf Schlegel findet sich so lediglich der Eintrag «Arnold Schlegel, Dürmentingen, Zeugniß 2. Claße»: vgl. UAT 60/1, Liber chirurgorum, S. 556. 
Dass Fridolin Schlegel seinen Sohn den Wundarztberuf ergreifen liess, muss insofern überraschen, als die Aussichten der nichtakademischen Chirurgen in den 1860er Jahren vergleichsweise schlecht waren. So hatten die 〈Handwerkschirurgen〉 zwischen 1814 und 1858 wesentliche Kompetenzbeschneidungen erfahren, die bereits nachhaltige Auswirkungen auf das Berufsbild und die wundärztliche Tätigkeit zeigten: 1814 hatte man in Württemberg zunächst neben der Aufhebung der chirurgischen Zunftladen vier unterschiedlich qualifizierte Klassen nichtakademischer Chirurgen gebil$\operatorname{det}^{8}$. Während die <Wundärzte I. Klasse〉 zu grossen chirurgischen Verrichtungen berechtigt waren, wurden die Vertreter der II. Klasse von schwierigen Operationen mit dem Messer ausgeschlossen. 〈Wundärzte III. Klasse〉 waren lediglich zu leichteren chirurgischen Behandlungen zugelassen. Der IV. und letzten Klasse gestand man lediglich Badergeschäfte zu.

Mit dem Jahr 1830 wurden nur noch 3 <Abteilungen〉 von Wundärzten mit teilweise stark eingeschränkten Arbeitsbereichen ausgebildet ${ }^{9}$. Die Badertätigkeit wurde nunmehr an Orten ohne Chirurgen IV. Klasse freigegeben.

1858 erfolgte auch die Aufhebung der 3. Abteilung. Die Wundärzte der 1. Abteilung mussten fortan auch die innere Medizin studiert haben und wurden 〈höhere〉 Chirurgen genannt; die Vertreter der 2. Abteilung bildeten die Klasse der $\langle\text { niederen Wundärzte }\rangle^{10}$.

Während in Preussen bereits 1852 das gesetzliche Aus für den Wundarztberuf verfügt worden war, wurden die «niederen〉 Chirurgen mit Rechtskraft der Deutschen Gewerbeordnung vom 1. Januar 1872 auch in Württemberg als letztem deutschen Einzelstaat - auf den «Aussterbeetat〉 gesetzt. 1872/73 wurden hier die letzten Wundarztprüfungen durchgeführt; in der Folgezeit wurden nur noch akademische Chirurgen ausgebildet ${ }^{11}$.

Als 1870 in Württemberg der Wundarztberuf zur Diskussion gestellt wurde, versah der 20jährige Schlegel gerade seinen Dienst als Feldunterarzt im Deutsch-Französischen Krieg. Dabei dürfte er wesentliche praktische Er-

8 Vgl. Hauptstaatsarchiv Stuttgart (i. f. z. als HStAS) E 10, Bü 35 (Bericht vom Ministerium des Innern vom 3.7.1813) sowie E 146 (2) alt, Bü 146 (Aufhebung der Zunftverfassung der Wundärzte und Bader). Weiterhin: General-Verordnung, die Aufhebung der Zunftverfassung der Wundärzte betreffend. Königlich-Württembergisches Staats- und RegierungsBlatt (i. f. z. als Reg.Bl) 1814, S. 173-178; StAL E 162 I, Bü 463, Schriftstück (i. f. z. als SSt) 5 .

9 Verordnung vom 14. Oktober 1830, betreffend die Abstufung in der Ermächtigung zur Ausübung der Wundarzneikunde. Reg.Bl 1830, S. 443-457, zur Vorgeschichte vgl. etwa HStAS E 151/51, Bü 152, insb. SSt 22 u. 25.

10 Königliche Verordnung, betreffend die Ermächtigung zur Ausübung der Wundarzneikunde vom 14. März 1858. Reg.Bl 1830, S. 70f; zur Vorgeschichte vgl. HStAS E 151/51, Bü 153.

11 Vgl. Staats-Anz Württemberg, 1.4. 1873, Nr. 76, S. 517; siehe auch StAL E 162 I, Bü 612 sowie UAT $60 / 1$. 
fahrungen in der Chirurgie gesammelt haben ${ }^{12}$. Nach seiner Militärentlassung im Jahr 1871 schrieb er sich - zusammen mit 36 Kollegen - noch im Wintersemester 1871/72 als Hospitant für das Fach «Niedere Chirurgie» an der Universität Tübingen ein ${ }^{13}$. Dort studierte er bis einschliesslich Sommersemester 1872 die Wundarzneikunde. Am 27. Mai 1872 wurde Schlegel - ebenfalls in Tübingen - als Wundarzt 2. Abteilung geprüft ${ }^{14}$. Sein wundärztliches Examen verdient aus mehreren Gründen Beachtung: Zum einen ist festzuhalten, dass Schlegel mit 22 Jahren zu den jüngsten Prüflingen zählte ${ }^{15}$.Zum anderen gehörte er zu den letzten im Deutschen Reich approbierten «Handwerkschirurgen ${ }^{16}$.

Das wundärztliche Prüfungszeugnis Schlegels gibt Aufschluss über seine fachliche Qualifikation. Hiernach zeigte er insgesamt «ziemlich gute Kenntnisse ..., auch einige Erfahrung in chirurgischen Operationen und auch einige eigene Erfahrung» ${ }^{17}$. In vier der neun Prüfungsfächer, namentlich in der chirurgischen Anatomie, der medizinischen Zeichenlehre und Therapie, der Arzneimittellehre sowie der Schutzpockenimpfung wies Schlegel 〈gute〉, in den übrigen Disziplinen (Physiologie, Pathologie, Nosologie, Operations- und Verbandslehre sowie gerichtliche Chirurgie) <ziemlich gute〉 Kenntnisse auf. Ein Vergleich mit anderen Prüflingen des Jahres 1872 zeigt, dass Schlegels Bewertungen überdurchschnittlich ausfielen. Wie die meisten Wundärzte legte er - ebenfalls 1872 - eine zusätzliche Prüfung zum Geburtshelfer $a b^{18}$. Insgesamt waren im Jahr 1872 in Württemberg noch 644 Wundärzte tätig, wovon $244 \mathrm{im} 1848$ gegründeten «Verein der württembergischen Wundärzte und Geburtshelfer» organisiert waren. Die Zahl der württembergischen Ärzte betrug zum gleichen Zeitpunkt $498^{19}$.

Offensichtlich hatte sich Schlegel von der Aufhebung des Berufsstandes eine grosszügige Übergangslösung für die noch verbliebenen «Handwerkschirurgen〉 erhofft, denn in späteren Jahren nahm er in einzelnen Schriften wiederholt auf jene Zeit Bezug. Dabei bezeichnete er die «Aufrechterhaltung und fortgesetzte Verengung der veralteten Schranken und Befugnissgrenzen für die Wundärzte ... um so unbilliger als im Nachbarstaate Oesterreich alle 3 Klassen von Wundärzten ... und auch in Preussen die sehr ver-

12 Vgl. Z Wundärzte Geburtsh 70,1919, S. I.

13 Vgl. Verzeichnis der Beamten, Lehrer und Studierenden der königlich württembergischen Universität Tübingen in dem Winter-Halbjahr 1871-72, Tübingen 1872, S. 19f; vgl. auch den handschriftlichen Eintrag Schlegels im Hospitantenbuch: UAT 5/39, 1871.

14 UAT $62 / 12$ a, 15.

$15 \mathrm{Vgl}$. UAT 5/39 (Hospitantenbuch).

16 Vgl. UAT 60/1 (Prüfungsbuch).

17 Wie Anm. 16.

18 UAT $62 / 10,82$.

19 Vgl. Medizinalber Württembg 1912, S. 16 sowie Z Wundärzte Geburtsh 25, 1872, S. 302. 
schieden qualifizierten Wundärzte I. Klasse - als Aerzte diplomiert wurden und sogar die preus. Wundärzte II. Klasse das unumschränkte freie interne Ordinationsrecht (auch bei inneren Krankheiten) besitzen und die württb. Wund- und Hebärzte einen Vergleich mit diesen nicht zu scheuen brauchen $»^{20}$.

Statt einer Befugniserweiterung hatte das Reichs-Impfgesetz vom 8. 4. 1874 weitere Restriktionen zur Folge, indem den württembergischen «Handwerkschirurgen> die Erlaubnis zur Schutzpockenimpfung entzogen wurde ${ }^{21}$. Auch die Anwendungsbeschränkung für Arzneimittel wurde wundärztlicherseits mit Kritik aufgenommen ${ }^{22}$.

Die Unzufriedenheit mit den Berufsbedingungen veranlasste Schlegel, frühzeitig berufspolitisch aktiv zu werden. Dass er bereits 1874 - im Alter von erst 24 Jahren - der fünfköpfigen «Vertrauenskommission der württembergischen Wundärzte II. Abteilung» angehörte, ergibt sich aus einer Petition, welche jenes Gremium am 21.12.1874 an das Innenministerium richtete. Ziel der Wundärzte war hiernach die «Regulierung ihrer beruflichen Wirksamkeit in Bezug auf die Anordnung und den Verkauf ihrer Medikamente, in Bezug auf die Armenpraxis und die Leichenschau» sowie die «Abänderung der Medizinaltaxe zur endlichen und geneigtesten Erledigung $»^{23}$. Im selben Jahr nahm Schlegel zudem erstmals an der Jahresversammlung des «Vereins der württembergischen Wundärzte und Geburtshelfer» teil ${ }^{24}$.

Bereits im Frühjahr 1875 richtete Schlegel zusammen mit dem Kornwestheimer Wundarzt Wacker eine weitere Petition an die Ständeversammlung, die auf eine den Ärzten vergleichbare Befugniserweiterung abzielte ${ }^{25}$. Schlegels berufspolitisches Engagement muss in den eigenen Reihen Beifall gefunden haben, denn im Rahmen der Vereinsversammlung vom 5. Juli 1875 wurde er zusammen mit zwei arrivierten Vereinsmitgliedern - dem Vorsitzenden der wundärztlichen Unterstützungskasse, Heinrich Koch von Fellbach, und dem Oberamtswundarzt Büchelen aus Marbach - gewählt, «um die nöthigen Schritte zu thun, die Standesinteressen zu wahren $»^{26} .1876$ richtete Schlegel - diesmal zusammen mit dem Wundarzt Baumgärtel aus Wald-

20 A. Schlegel, Stellung und Verhältnisse der württembergischen Wund-Aerzte und Geburtshelfer (II.Abteilung) in ihrer geschichtlichen Entwicklung und im Vergleiche mit den Nachbarstaaten, Fellbach 1894, S. 1.

21 Wie Anm. 20, S. 6; vgl. auch Z Wundärzte Geburtsh 25, 1872, S. 159 u. 234.

22 vgl. Z Wundärzte Geburtsh 25, 1872, S. 55f.

23 HStAS E 151/51, Bü 154, ad SSt 182.

24 Vgl. Z Wundärzte Geburtsh 26, 1875, S. 124.

25 HStAS E 151/51, Bü 154, ad SSt 83 sowie StAL E 162 I, Bü 459, SSt 76-78.

26 Z Wundärzte Geburtsh 26, 1875, S. 197. 
dorf - im Auftrag des Vereins eine Eingabe an den Bundesrat in Berlin, die eine Erweiterung der Rechte zur Verordnung von Arzneimitteln, die Gleichstellung des Vereins mit den Organisationen der Ärzte, Tierärzte und Apotheker, die Besorgung der Leichenschau und die Einreihung der Wundärzte im Militär als «Unterärzte» zum Ziel hatte ${ }^{27}$.

Auch in Schlegels Privatleben brachte das Jahr 1875 eine Veränderung. Offensichtlich fand er im dörflichen Dürmentingen kein hinreichendes Auskommen, denn er liess sich nunmehr in Altshausen/Oberamt Saulgau nie$\operatorname{der}^{28}$. Neben seiner interessenpolitischen Tätigkeit verfasste er nunmehr auch fachliche Beiträge. So erschien 1876 in der «Zeitschrift für Wundärzte und Geburtshelfer», dem Organ des «Vereins der württembergischen Wundärzte und Geburtshelfer», ein mehrteiliger Übersichtsartikel über Aneurysmen ${ }^{29}$. Bereits 1878 wurde an gleicher Stelle ein ähnlich umfassender Beitrag zu den Lageveränderungen und zur Exstirpation des Uterus veröffentlicht. Im selben Jahr erfolgten zudem in Altshausen seine Heirat mit der Schweizerin Rosa Fuchs sowie die Geburt seines einzigen Kindes Rosa Sophie ${ }^{30}$. Bald darauf trat Schlegel mit der Ankündigung hervor, ab 1881 mit Hilfe des Vereins einen wundärztlichen «Medicinal-Kalender» herausgeben zu wol$l^{3}{ }^{31}$. Nachdem jener Kalender mit Unterstützung des Vereinsvorstandes mehrmals in der «Zeitschrift für Wundärzte und Geburtshelfer» angekündigt worden war, zog Schlegel schliesslich sein Projekt wieder zurück, indem er ausführte, die Zeit der Vorbereitung sei nicht ausreichend gewesen. Gleichzeitig dankte er den «Herrn Pränumeranden» für ihr Interesse ${ }^{32}$. Die Ursache für die Stornierung des Kalenders war möglicherweise die Entscheidung, Altshausen wieder zu verlassen und in die Schweiz überzusiedeln.

1881 liess sich die Familie Schlegel in Einsiedeln, dem Herkunftsort von Rosa Schlegel, nieder ${ }^{33}$. Kurze Zeit später kehrte man jedoch wieder nach Württemberg zurück. Dies geht nicht nur aus einem diesbezüglichen «Heimatschein» im Stadtarchiv Einsiedeln hervor, sondern ist auch aus einer Notiz der Vereinszeitschrift ersichtlich ${ }^{34}$. Schlegels Aufenthalt im oberschwäbischen Aulendorf war wiederum nur von kurzer Dauer. Während er am 2. Juli

27 HStAS E 151/51, Bü 154, ad SSt 195 u. 196.

28 Z Wundärzte Geburtsh 26, 1875, S. 224.

29 Z Wundärzte Geburtsh 27, 1876, S. 3, 97, 189, 285.

30 Bürgermeisteramt Dürmentingen, Familienregister, Bd. 2, Bl. 222 u. Bd. 4, Bl. 11.

31 Z Wundärzte Geburtsh 31, 1880, S. 189 u. 303.

32 Ebd., S. 383.

33 Bezirksarchiv Einsiedeln (i.f.z.als BAE), Einwohnerkontrolle 1881 (Zuzug vom 5.5.1881); vgl. auch Z Wundärzte Geburtsh 32, 1881, S. 96.

34 Z Wundärzte Geburtsh 34, 1833, S. 277. 
1883 auf der Jahresversammlung des Vereins in Biberach noch mit dem Wohnort «Aulendorf» geführt wurde, war er seit dem 20.12.1883 wieder als Einwohner Einsiedelns registriert ${ }^{35}$. Auch in der ZWG ist die neuerliche Auswanderung in die Schweiz vermerkt; gleichzeitig findet sich eine Notiz, wonach Schlegel sich nunmehr als «Gastgeber zum Bären» empfehle ${ }^{36}$. Dass es sich hierbei nicht um eine kurzfristige oder nebenberufliche Tätigkeit handelte, belegen Akten des Stadtarchivs Einsiedeln. So finden sich für den Zeitraum zwischen 1886 und 1922 wiederholt Dokumente, die Schlegel als Pächter des Gasthofs zum Bären ausweisen ${ }^{37}$. Hinweise auf eine Tätigkeit in seinem erlernten Beruf als Wundarzt sind dagegen nicht auszumachen. In den kantonalen Akten findet sich keine Bewilligung für eine Behandlertätigkeit $^{38}$. Schlegel selbst gibt verschiedentlich an, sich in der Schweiz auf die Zahnheilkunde spezialisiert zu haben und bezeichnet sich als <eidgenössischen Zahnarzt ${ }^{39}$.

Die Tatsache, dass Schlegel über einen Zeitraum von nahezu 4 Jahrzehnten in Einsiedeln den fachfremden Beruf eines Wirts ausübte, mag auf den ersten Blick überraschen. Hintergrund dieser Entscheidung dürfte jedoch die Tatsache gewesen sein, dass ihm die chirurgische Tätigkeit kein gesichertes Auskommen ermöglichte. Dies geht aus späteren Schriften Schlegels her$\operatorname{vor}^{40}$.

Aus offiziellen Medizinalstatistiken ergibt sich, dass Schlegel keinesfalls der einzige württembergische Wundarzt war, der sich als Wirt betätigte. So wurden bereits 1872 im Königreich Württemberg 24 Wundärzte registriert, die im Nebenberuf eine Gastwirtschaft betrieben. Ingesamt übten 1872 16 Prozent aller württembergischen Zivilwundärzte, d.h. 103 von insgesamt 641 Personen, eine Nebentätigkeit aus ${ }^{41}$. Bis 1880 stieg die Zahl der Wundärzte mit amtlich registrierter Nebenerwerbsquelle auf 21 Prozent an. Damit gingen 98 von 469 verbliebenen Wundärzten einer Zweitbeschäftigung nach. Unter den betroffenen Wundärzten befanden sich wiederum 15 Wirte ${ }^{42}$.

35 BAE, Kontrolle der niedergelassenen Ausländer, Heimatschein vom 12. März 1881, Eingang 20.12. 1883.

36 Z Wundärzte Geburtsh 34, 1833, S. 391.

37 Zu den diesbezüglichen Wirtschaftspatenten vgl. Amtsbl Kanton Schwyz 1886, S. 251; 1900, S. $69 ; 1904$, S. $295 ; 1922$, S. 115 u. 529.

38 Schriftliche Auskunft von Hr. E. Müller, Bezirksverwaltung Einsiedeln, vom 24. 11. 1995.

39 Vgl. StAL E 162 II, Bü 1482 (Personalakte Arnold Schlegel), SSt 4.

40 Ebd., SSt 2.

41 Medizinalber Württembg 1872, S. 61.

42 Medizinalber Württembg 1879-1881, S. 79. 
Demgegenüber belief sich der offizielle Anteil der Wundärzte mit Nebenberuf 1890 bereits auf 25 Prozent $^{43}$. Der steigende Anteil der Wundärzte mit amtlich registrierter Nebentätigkeit lässt den Schluss zu, dass sich die finanzielle Lage der Wundärzte im Beobachtungszeitraum zunehmend verschärfte.

Obgleich die Tätigkeit Schlegels als Wirt offensichtlich keine Ausnahme darstellte, ist zu beachten, dass es sich bei dem «Bären» um einen sehr bekannten Gasthof handelte, dessen Tradition bis ins Mittelalter zurückreicht und der bis auf den heutigen Tag als «Zunfthaus und Hotel Bären am Klosterplatz» eine zentrale Rolle im Ortsbild Einsiedelns spielt ${ }^{44}$. Bei der Übernahme des Gasthofs dürften familiäre Beziehungen eine Rolle gespielt haben, denn in einer Hauschronik des «Bären» wird der Bruder Rosa Schlegels, Josef Meinrad Fuchs-Brügger, als «Sohn des Bärenwirts» bezeichnet. Ebenda findet sich auch der Hinweis, Arnold Schlegel sei «um die Jahrhundertwende ...Wirt auf dem Bären» gewesen ${ }^{45}$. Dass Rosa Schlegel einer gutsituierten Familie entstammte, beweist die Tatsache, dass Josef Meinrad Fuchs-Brügger Medizin studieren konnte und praktischer Arzt wurde ${ }^{46}$. Schlegel selbst gab an, dass es finanzielle Unwägbarkeiten seien, die ihn von der Rückkehr in die Heimat abhielten ${ }^{47}$. Stattdessen nahm er in den nachfolgenden 4 Jahrzehnten immer wieder zeitraubende und kostspielige Reisen nach Württemberg in Kauf, um seine standespolitischen Ambitionen vor Ort weiterzuverfolgen. Ferner lässt der Umstand, dass Schlegel den «Verein der württembergischen Wundärzte und Geburtshelfer» in den nachfolgenden Jahren wiederholt finanziell unterstützte, auf ein gesichertes Auskommen in der Schweiz schliessen ${ }^{48}$.

Wie die wiederholte Ausstellung von Heimatscheinen belegt, kehrte Schlegel auch in den nachfolgenden Jahren regelmässig - zumeist für einige Wochen - nach Württemberg zurück ${ }^{49}$. Offensichtlich war er mit seiner beruflichen Situation jedoch nicht zufrieden. Dies zeigt ein Antrag Schlegels aus dem Jahr 1887 auf Zulassung zum medizinischen Studium an der Universität Tübingen. Schlegels Gesuch wurde abgewiesen mit dem Hinweis, dass die Aufnahme des Medizinstudiums an die Maturität gebunden sei ${ }^{50}$.

43 Medizinalber Württembg 1888-1890, S. 106.

44 W. Kälin, Zunfthaus und Hotel Bären Einsiedeln am Klosterplatz. Eine kleine Hauschronik, Einsiedeln o. J., S. 7.

45 Wie Anm. 44.

46 Vgl. Zivilstandesamt Einsiedeln, Eintrag vom 2. 6.1901.

47 Vgl. StAL E 162 II, Bü 1482, SSt 2.

48 Vgl. Z Wundärzte Geburtsh 49, 1898, S. 264f. sowie 64, 1913, S. 35.

49 BAE, Kontrolle der niedergelassenen Ausländer.

50 Ebd., SSt 1. 
Seiner Publikationstätigkeit tat dieser Bescheid allerdings keinen Abbruch. Zwischen 1891 und 1894 erschienen im Vereinsorgan weitere Artikel von Schlegel über die Immunität gegen Infektionskrankheiten, über die Diphtherie, über Gaumendefekte und «Velostaphyloraphie〉 sowie über Asphyxia Neonatorum ${ }^{51}$.

Am 2. Juli 1891 wurde ein Entwurf des deutschen Bundesrats zur Arzneimittelfrage bekannt, den Schlegel als schweren «Schlag gegen die (württbg.) Wundärzte» wertete. Der entsprechende württembergische Ministerialerlass vom 19. Dezember 1891 untersagte aus Sicht der Chirurgen eine Reihe «unentbehrlicher Anwendungsarten». Andere Medikamente würden dagegen «dem gesamten Laientum zum äusserlichen Gebrauche freigegeben ${ }^{52}$.

Anlässlich der Vereinstagung des Jahres 1892 in Stuttgart führte Schlegel zusammen mit dem Vorsitzenden Baumgärtel die standespolitische Diskussion. Dabei wurde eine Eingabe an den Innenminister «um Abhilfe verschiedener Misstände» beschlossen; zudem sprach man einen «Toast» auf Schlegel aus, «welchen der Eifer für unsere Sache von den schönen Bergen der Schweiz in Schwabens Residenzstadt getrieben» habe ${ }^{53}$. In der Ausgabe der Vereinszeitschrift desselben Jahres veröffentlichte Schlegel zudem einen kritischen standespolitischen Beitrag mit dem Titel «Vom Baume der Erkenntniss $»^{54}$.

1894 erschien Schlegels erste Monographie über die Stellung der württembergischen Wund- und Hebärzte. Es handelte sich um eine 17seitige historische und berufspolitische Abhandlung in Folioformat. Schlegel erhob in seiner Darstellung die Forderung, den approbierten Wund- und Hebärzten «nach so vieljähriger praktischer Erfahrung das Recht» zuzugestehen, «alle nötigen Mittel auch innerlich verordnen zu dürfen». Auch sollten die «letzten Veteranen des so rasch aussterbenden Standes württ. Wundärzte und Geburtshelfer ... etwas mehr als bisher als Aerzte angesehen werden $»^{55}$. Im gleichen Jahr betonte Schlegel auf der Vereinsversammlung in Ulm, «dass er schon seit Jahrzehnten verlange, die Wund-Aerzte sollten sich doch den mit der Approbation verbundenen $\S 8$ der Allerh. Verordnung vom 14. Oktober 1830 nicht so mir nichts dir nichts entziehen lassen». Jener Paragraph beinhaltete das Recht, in dringenden Fällen «jede innerliche und äusserliche

51 Z Wundärzte Geburtsh 42,1891, S. 225 u. 321; 44, 1893, S. 26, 94, 208; 45, 1894, S. 68, 140, 237, 334.

52 Wie Anm. 20, S. 7.

53 Z Wundärzte Geburtsh 43, 1892, S. 228 u. 229 (Zitat).

54 Z Wundärzte Geburtsh 43, 1892, S. 1.

55 Wie Anm. 20., insb. S. 17 (Zitate). 
Hilfe zu leisten», was auch das Verordnungsrecht für alle Arzneimittel einschliesse $^{56}$. Auf Antrag Schlegels wurde beschlossen, «Seiner Majestät schriftlich oder mündlich - oder in beiden Arten - die Angelegenheiten zu unterbreiten, um, was durch den Buchstaben des Rechts nicht zu erlangen, durch Allerhöchste Gnade zu erreichen ${ }^{57}$. In der gleichen Sitzung beklagte Schlegel, dass es lange versäumt worden sei, «in höheren und höchsten Regionen daran zu erinnern, dass wir auch noch da und weder mund- noch ganz tot seien und ebenso brave Landeskinder wie andere, denen mehr Recht und Gunst zu Teil werde ${ }^{58}$.

Schlegel liess seinen umjubelten Worten Taten folgen, denn am 9.1.1895 wurde der Wahlschweizer zusammen mit dem Vereinsvorsitzenden Baumgärtel von König Wilhelm II. von Württemberg empfangen, wobei «sich auch S. Majestät eingehend für die Sache interessierte und Unterstützung vorgetragener Bitten gnädigst zusagte $»^{59}$. Am 22. und 25. 1. 1895 fanden weitere diesbezügliche Besprechungen zwischen Schlegel, Baumgärtel und Mitgliedern des Medizinalkollegiums sowie mit Innenminister von Pischek statt ${ }^{60}$. Am 7. August des gleichen Jahres verfasste Schlegel zudem eine Bittschrift an von Pischek, in welcher er die Ziele der Wundärzte erneut in Erinnerung rief und «in Zweifelsfällen einen allerhuldvollsten Gnaden-Akt Seiner Majestät» erbat ${ }^{61}$. Im Rahmen der Versammlung des «Vereins der württembergischen Wundärzte und Geburtshelfer» vom 1. Juli 1895 wurde Schlegel ein besonderes Lob zugesprochen, da dieser «dem Vereinsorgane schon manche instructive Arbeiten überwies und vor allem durch seine selbstlose und uneigennützige Thätigkeit zur Besserstellung unseres Standes und Berufes sich den Dank der Collegen» verdient habe ${ }^{62}$. Zugleich wurde Schlegel zum Ehrenmitglied des Vereins ernannt ${ }^{63}$. Auf derselben Tagung stellte Schlegel den Antrag, zu gegebener Zeit eine Anfrage an den Bundesrat zu richten, inwieweit die Wundärzte als Ärzte anzusehen seien ${ }^{64}$.

Im darauffolgenden Jahr trat Schlegel erneut mit einer standespolitischen Initiative hervor. Hintergrund jener Massnahme war die Tatsache, dass in

56 Z Wundärzte Geburtsh 45, 1894, S. 274-283, insb. S. 279f. Zum Wortlaut des betreffenden Paragraphen vgl. V.A. Riecke, Das Medizinalwesen des Königsreichs Württemberg, unter systematischer Zusammenstellung der dasselbe betreffenden Gesetze, Verordnungen, Verfügungen, Normalerlasse, Stuttgart 1856, S. 76.

57 Z Wundärzte Geburtsh 45, 1894, S. 280.

58 Ebd., S. 281.

59 Z Wundärzte Geburtsh 59, 1908, S. 135.

60 Wie Anm. 59.

61 HStAS E 151/51, Bü 154, SSt 256.

62 Z Wundärzte Geburtsh 46, 1895, S. 281.

63 Ebd., S. 290.

64 Ebd., S. $280 f$. 
〈Börner's Reichs-Medizinalkalender〉 alle Wundärzte mit Ausnahme der württembergischen aufgeführt waren ${ }^{65}$. Nachdem keine Aufnahme in das Börnersche Werk erreicht werden konnte, veröffentlichte Schlegel mit Unterstützung des Vereins ein 33seitiges «Verzeichnis der württembergischen Wundärzte und Geburtshelfer, nach dem Stande vom 1. Januar 1896». Das Vorwort zu jener Abhandlung verfasste derVereinsvorsitzende Baumgärtel ${ }^{66}$.

Neben berufspolitisch motivierten Petitionen und Publikationen wandte sich Schlegel 1896 aber auch mit einer Reihe persönlicher Schreiben an staatliche Instanzen. Gegenstand jener Schriften, von denen fünf im Staatsarchiv Ludwigsburg überliefert sind, war die Bitte um Erlaubnis zur Führung des chirurgischen Doktortitels ${ }^{67}$. Schlegel hatte von einem Medical College in Chicago - angeblich für seine wissenschaftlichen Publikationen in der «Zeitschrift für Wundärzte und Geburtshelfer» - in absentia den Dr. med. et chir. honoris causa erworben und bat nunmehr in einzelnen Schreiben an das Medizinalkollegium und an Regierungsdirektor von Gessler um die Anerkennung des ausländischen Titels. Den Erwerb jenes amerikanischen Diploms rechtfertigte er mit der Tatsache, dass für ihn in Deutschland keine Promotionsmöglichkeit bestanden habe. Andererseits sei er jedoch überzeugt, «dass auch deutschen Fakultäten die vorgelegten Arbeiten zur Promotion genügen würden, wenn diejenigen Vorbedingungen erfüllt werden könnten, die in Amerika zur Zeit noch nicht verlangt werden ${ }^{68}$. Wann Schlegel den ausländischen Ehrentitel erworben hatte, geht aus den Dokumenten nicht eindeutig hervor. Allerdings spricht einiges dafür, dass es sich hierbei um das Jahr 1893 handelte. Schlegel fügt einem seiner Bittschreiben als «Arbeitsprobe` seine Abhandlung über 〈Velostaphylorhaphie〉 bei. Jene Arbeit wurde 1893 in der «Zeitschrift für Wundärzte und Geburtshelfer» publiziert; in derselben Ausgabe wird Schlegel zudem erstmals mit dem Doktortitel geführt ${ }^{69}$.

Die Bittgesuche Schlegels entbehren nicht einer gewissen Pikanterie, denn sie erfolgten auf dem Hintergrund eines weitreichenden Handels mit ausländischen Diplomen. Vor allem seit der Mitte der 1870er Jahre war die Zahl <amerikanischer Doktoren in Deutschland stark angestiegen. Im gleichen Masse vermehrten sich die Schwindelinstitute in den USA, die nur äusserlich dem Anspruch gerecht wurden, einen Lehrauftrag zu erfüllen ${ }^{70}$. Dem In-

65 Wie Anm. 20, S. 9; vgl. auch StAL E 162 I, Bü 459, SSt 120, 127, 131.

66 A. Schlegel. Verzeichnis der württembergischen Wundärzte und Geburtshelfer nach dem Stande vom 1. Jan. 1896. Fellbach 1896 (vgl. auch StAL E 162 I, Bü 459, SSt 131).

67 StAL E 162 II, Bü 1482, SSt 1-5.

68 Vgl. StAL E 162 II, Bü 1482, SSt 1.

69 Z Wundärzte Geburtsh 44, 1893, S. IV.

70 Zum Niveau amerikanischer «Medical Schools» im vergangenen Jahrhundert vgl. 
teressenten blieb oft sogar die Anreise in die USA erspart; man ermöglichte es ihm, den Titel in absentia - und damit ohne irgendeinen Zeitverlust - zu erwerben. Zahnärztlicherseits wurden Institute beschrieben, die den Titel 〈Doctor honoris causa〉 an Zahnärzte und vor allem Zahnkünstler zu Preisen zwischen 12 und 150 Dollar verkauften ${ }^{71}$. Als am 26.5.1896 das «Reichsgesetz zur Bekämpfung des unlauteren Wettbewerbs> verabschiedet wurde, fand dieses auch in der Titelfrage Anwendung ${ }^{72}$. Die restriktive Haltung des Gesetzgebers trug schliesslich dazu bei, dass die Führung amerikanischer Titel in der Folgezeit nur noch selten erlaubt wurde.

Besonders schwer nachzuvollziehen war im Fall Schlegels die Tatsache, dass diesem nicht etwa nur der Titel eines 〈Dr. chir〉., sondern zugleich der Grad des «Dr. med.» zugesprochen worden war. Schlegel selbst versuchte diese Unstimmigkeit mit dem Hinweis zu entschärfen, dass das Institut in Chicago keinen reinen Chirurgentitel vergäbe; dessen ungeachtet ziele seine Bitte nur auf die Gewährung des chirurgischen Titels «unter selbstverständlichem Verzicht auf den medizinischen ${ }^{73}$. Um die praktische Bedeutung seines Gesuchs zu untermauern, führte Schlegel an, dass er die Absicht habe, in seine deutsche Heimat zurückzukehren. Zur Rechtfertigung seiner Bitte bezog er sich im weiteren auf Vorschriften der Universität Zürich, wo «alle Promotionen ... lediglich auf Grund der eingereichten Dissertation stattfinden u. deshalb die Erlangung ausländischer Diplome unter denselben Bedingungen nichts ungewöhnliches in der Schweiz» sei. Auch hätten einzelne seiner Arbeiten «nach dem Urteil von Professoren selbst an deutschen Universitäten genügt». Zudem seien in Württemberg keine Wundärzte I. Klasse mit dem Titel eines Dr. chir. mehr nachzuweisen, so dass «sowohl die Absicht wie die Möglichkeit einer Verwechselung gänzlich ausgeschlossen» sei. Schliesslich handle es sich nur darum, «ohne Strafe fürchten zu müssen, bzw. um sich keiner Ueberschreitung schuldig zu machen, - in bescheidenster

T. Goebel, Ärzte und Rechtsanwälte in den USA 1800-1920. Der schwierige Weg zur Professionalisierung, in: GG 16, 1990, S. 318-342. Goebel beschreibt am Beispiel der Ärzte und Anwälte die vergeblichen Bemühungen der Vertreter freier Berufe um eine Standardisierung der Ausbildung.

71 C. Blochmann u. P. Steinberg, Der Handel mit amerikanischen Doktor-Diplomen und die «Institute» von C. A. Weil und Fritz Huxmann. Herausgegeben vom Vereinsbund Deutscher Zahnärzte, Berlin 1902, S. 6. Die Gründung jener Anstalten erfolgte zumeist in privater Initiative und «nach Antragstellung der Gründer ohne vorherige Prüfung ihrer Qualifikation». Zu den Hintergründen des Diplomschwindels im Bereich der Zahnheilkunde vgl. D. Gross, Die schwierige Professionalisierung der deutschen Zahnärzteschaft (1867-1919), Frankfurt a. M. 1994 (= Europäische Hochschulschriften, Reihe 3, 609), insb. S. 187-196.

72 Vgl. J.A.Zehnter, Die Führung zahnärztlicher Titel sowie des Doktortitels durch im Inland nicht approbierte Personen, Berlin 1906, S. 128 f.

73 StAL E 162 I, Bü 1582, SSt 4 (Zitat). 
Weise von dem Titel Gebrauch machen zu dürfen, da er auch sonst noch nie und nirgends auch nur die geringste Strafe erlitten hat und sich einer gerichtlichen Entscheidung deshalb nicht gern aussetzen möchte» ${ }^{74}$.

Im Gegensatz zu den Beteuerungen Schlegels lässt sich eindeutig nachweisen, dass dieser durchaus vor jenem Schreiben unerlaubterweise den Doktortitel geführt hatte. So verfasste Schlegel das Protokoll über die Jahresversammlung des «Vereins der württembergischen Wundärzte und Geburtshelfer» in Ulm am 2. Juli 1894 und führte sich selbst in der Teilnehmerliste, im Unterschied zu allen anderen Anwesenden, als «Dr. Schlegel» auf ${ }^{75}$.

Das letztdatierte der fünf überlieferten, privaten Schreiben von Schlegel enthält die Mitteilung, dass er sein Gesuch zurückziehe und persönlich vorstellig werden wolle, «da er unvorhergesehener Weise veranlasst ist eine Reise nach Deutschland zu machen wobei er Stuttgart berühren u. sich die Ehre geben wird Euer Hochwohlgeboren seine ergebenste Aufwartung zu machen». Einem handschriftlichen Vermerk zufolge erschien Schlegel am 17. Oktober 1896 «persönlich auf dem Sekretariat, wobei ihm die vorgelegten Akten wieder ausgehändigt wurden $»^{76}$. Ob es behördlicherseits zu einer Anerkennung des Dr.-Titels kam, geht aus den Archivalien nicht eindeutig hervor. Höchstwahrscheinlich kam es zu keiner Genehmigung, denn auch in einer Akte des Medizinalkollegiums aus dem Jahr 1908 ist lediglich von dem «Wundarzt Schlegel» die Rede ${ }^{77}$. Selbst in seinen seit 1894 veröffentlichten Monographien führt Schlegel den fraglichen Doktortitel nicht $a^{78}$. Demgegenüber lassen sich in der Vereinszeitschrift und unter den Akten des Bezirksarchivs Einsiedeln in den nachfolgenden Jahren immer wieder Hinweise auf $<$ Dr. (Arnold) Schlegel finden ${ }^{79}$.

1897 trat Schlegel auf der Jahresversammlung des Vereins in Ulm, wie in den Vorjahren, als Referent hervor. Zudem wurde er - ungeachtet seines Schweizer Wohnsitzes - in den Vereinsvorstand berufen ${ }^{80}$. Im Vereinsorgan desselben Jahres erschienen darüber hinaus mehrere wissenschaftliche Artikel Schlegels ${ }^{81}$ sowie ein standespolitischer Beitrag über «das neue Ordinationsrecht der Wundärzte und Geburtshelfer», in welchem er zu der Schlussfolgerung gelangt, dass die wiederholten Petitionen und Audienzen zu einer Besserstellung der Wundärzte geführt habe: «so leuchtet doch endlich noch

74 StAL E 162 I, Bü 1582, SSt 2 (Zitate).

75 Z Wundärzte Geburtsh 45, 1894, S. 274-283, insb. S. 275.

76 StAL E 162 I, Bü 1582, SSt 5.

77 HStAS E 151/51, Bü 154, SSt 315.

78 Vgl. etwa Anm. 20, 66 u. 84.

79 Vgl. etwa Z Wundärzte Geburtsh 70,1919, S. 49.

80 Z Wundärzte Geburtsh 48, 1897, S. 276.

81 Ebd., S. 68 u. 230. 
ein freundlicher Stern über dem düstern Lebensabend des aussterbenden Standes $»^{82}$.

Die Versammlung des Jahres 1898 stand ganz im Zeichen des 50jährigen Vereinsjubiläums. Aus ebendiesem Anlass hielt Schlegel einen Vortrag «Über die Entwickelung der Heilkunde und speziell der Chirurgie im 19. Jahrhundert in Deutschland». Sein Referat stiess laut Protokoll auf grosses Interesse und wurde mit einem «Toast» auf den Referenten aufgenommen. Überdies überreichte Schlegel jedem der 59 Anwesenden eine «gratis eingehändigte Festgabe zum 50jährigen Jubiläum des Vereins ${ }^{83}$. Schlegel überrascht in seiner dritten Monographie durch einen vergleichsweise versöhnlichen Ton. So kommentiert er die Erweiterung des Ordinationsrechts mit den Worten: «Für den wundärztlichen Stand, der sich endlich wieder eines grösseren Interesses und Wohlwollens von hoher Seite erfreuen darf, könnte dieser an sich bescheidene Erfolg bei einiger Toleranz der Herren Ärzte und hochgeneigter fernerer Rücksichtnahme auf die notwendigsten Existenzbedingungen doch ein befriedigender genannt werden. Er verpflichtet aber auch zu ehrerbietigster Dankbarkeit gegen die hohen Königlichen Behörden $»^{84}$. Das 44seitige Werk wurde wie die vorhergehenden in Fellbach gedruckt. Die Tatsache, dass Schlegel die Abhandlung kostenfrei verteilen liess, liefert einen weiteren Hinweis auf die finanzielle Unabhängigkeit des Wahlschweizers ${ }^{85}$.

Auch in den nachfolgenden Jahren blieb das berufspolitische Engagement Schlegels ungebrochen. Im Rahmen der Jahrestagung von 1899 sprach er «über Standesangelegenheiten ... und ... die Gefahren, welche unserem Stande von einer etwaigen Aufhebung der Gewerbefreiheit drohen». Dabei empfahl er, württembergische Reichstagsabgeordnete um Unterstützung zu bitten ${ }^{86} .1900$ erschien eine Abhandlung «Über den Einfluss des bürgerlichen Gesetzbuches auf die Ausübung der Heilkunde ${ }^{87}$. Im nachfolgenden Jahr referierte Schlegel über eine Eingabe der Wundärzte an den Landtag bezüglich ihrer Zulassung zur Kassenpraxis ${ }^{88}$. Schlegel hob hervor, dass die besagte Petition in den Kommissionen erörtert und gemeinsam mit der ärztlichen Eingabe um freie Arztwahl der Regierung übergeben worden sei. Er schloss

82 Ebd., S. 60 u. 307; zur Verfügung des Innenministeriums bezüglich der Arzneimittelabgabe vgl. Z Wundärzte Geburtsh 17, 1896, S. $309 f$.

83 Z Wundärzte Geburtsh 49, 1898, S. 222-238 u. S. 264 (Zitat).

84 A. Schlegel, Rückblicke auf die Geschichte des wundärztlichen Standes. Festgabe zum fünfzigjährigen Jubiläum des Vereins württembergischer Wundärzte und Geburtshelfer, Fellbach 1898, insb. S. 43 (Zitat).

85 Z Wundärzte Geburtsh 49, 1898, S. 264.

86 Z Wundärzte Geburtsh 50, 1899, S. 281-285, insb. S. 284.

87 Z Wundärzte Geburtsh 51,1900, S. 31.

88 Z Wundärzte Geburtsh 52, 1901, S. 174-180. 
seine Ausführungen mit dem Vorschlag, «eine Bitte an das Kgl. Ministerium $\mathrm{zu}$ richten, dass bei den Beamten der Oberämter unsere Stellung und Befugnisse mehr präcisiert werden» ${ }^{89}$.

Die Jahre 1902 bis 1907 sind ebenfalls durch eine Vielzahl fachlicher und standespolitischer Beiträge Schlegels gekennzeichnet ${ }^{90}$. Von besonderem Interesse ist eine Begebenheit aus dem Jahr 1908, die eine persönliche Beurteilung Schlegels durch das Medizinalkollegium zur Folge hatte. Hintergrund war das 60jährige Jubiläum des Vereins, das am 6. Juli des Jahres «als letztes Familienfest» des aussterbenden Standes in Stuttgart begangen werden sollte. Aus diesem Anlass brachte der Vereinsvorsitzende, Gustav Baumgärtel, seinen Kollegen Arnold Schlegel für die «Verleihung einer Auszeichnung» durch den König in Vorschlag. Die Bitte wurde vom Innenministerium an das Medizinalkollegium zur Stellungnahme weitergereicht. Das Medizinalkollegium begrüsste in einem Schreiben an das Ministerium vom 25. Juni 1908 die Idee, würdige Wundärzte auszuzeichnen, «um auf diese Weise dem Stand der Wundärzte eine Anerkennung und Freude zu Teil werden zu lassen ${ }^{91}$. Allerdings sprach man sich ebenso deutlich gegen eine Ehrung Schlegels aus. So konstatierte der Präsident des Kollegiums, er vermöge den Vorschlag, «den früher in Altshausen, seit längerer Zeit aber in Einsiedeln in der Schweiz als Verwalter eines dortigen Logierhauses ansässigen Wundarzt Schlegel» auszuzeichnen, «nicht zu unterstützen» ${ }^{92}$. An seiner Stelle wurden andere Wundärzte oder aber - zum wiederholten Male Baumgärtel als mögliche Empfänger von Ehrungen genannt.

Der Grund für die ablehnende Haltung des Medizinalkollegiums bleibt unerwähnt. Möglicherweise hatte sich Schlegel mit der Führung des fraglichen Doktortitels desavouiert; vielleicht war seine Hartnäckigkeit bei der Verfolgung standespolitischer Ziele in besagtem Gremium auf Kritik gestossen. Ebenso denkbar ist allerdings die Vorstellung, dass das Medizinalkollegium die Auszeichnung prinzipiell lieber einem in Württemberg ansässigen Wundarzt als einem «Logierhausverwalter» aus Einsiedeln zusprechen wollte.

Auf das Engagement Schlegels hatte jene Entscheidung jedoch keinen Einfluss, denn auch in den nachfolgenden Jahren ist der Name Schlegel immer wieder mit berufspolitischen Aktivitäten verbunden. 1908 erschien als Zeitschriftenbeitrag ein aktuelles, von ihm zusammengestelltes Verzeichnis

89 Ebd., S. 170 (Zitat).

90 Z Wundärzte Geburtsh 53,1902, S. 174; 54, 1903, S. 60 u. 128; 56, 1905, S. 168 u. 171; 58, 1907, S. 188.

91 HStAS E 151/51, Bü 154, SSt 315.

92 Wie Anm. 91. 
der württembergischen Wundärzte und Geburtshelfer ${ }^{93}$. Auf der Jubiläumsversammlung referierte Schlegel ferner über «Neue Krankheiten und ihre Behandlung $»^{94}$.

Auch im darauffolgenden Jahr war Schlegel aktiv. So rief er in der «Zeitschrift für Wundärzte und Geburtshelfer» zu einer regeren Vereinsarbeit auf und sprach über <Ulcera cruris und deren Behandlung ${ }^{95} .1910$ referierte Schlegel auf der Jahresversammlung über den Aderlass. In der Jahresausgabe der Vereinsschrift war er zudem mit zwei Fachbeiträgen und mit Nachrufen auf verstorbene Kollegen vertreten ${ }^{96} .1911$ wurde «die uneigennützige Collegialität Schlegels» im Rahmen der Jahresversammlung erneut hervorgehoben. Schlegel bringe «in geistiger und materieller Hinsicht dem Vereine Opfer ... ohne Nutzen davon zu haben» und ohne «alljährlich die Beschwerden und Kosten der langen Reise von der Schweiz heraus» zu scheuen ${ }^{97}$. Auch als Autor der Vereinszeitschrift war Schlegel erneut vertreten ${ }^{98}$.

Am 6. März 1913 überreichte Schlegel seinem Kollegen Baumgärtel eine Geldspende über 20 Mark «zur Bestreitung der höheren Druckkosten der Zeitschrift» ${ }^{99}$. Nur drei Monate später am 13.6.1913 starb die Ehefrau Schlegels in Einsiedeln ${ }^{100}$. Die besondere Stellung Schlegels unter den wundärztlichen Kollegen ist daran zu ersehen, dass Baumgärtel in seiner Ansprache zu Beginn der Vereinsversammlung vom 7. Juli auf den Tod Rosa Schlegels Bezug nahm, indem er auf ihr Engagement verwies: «Sie hat gewiss auch manches mit getragen, dessen hier und heute von uns dankbar gedacht werden soll» ${ }^{101}$. Das Ableben seiner Frau konnte Schlegel nicht davon abhalten, auf jener Tagung einen Vortrag zu halten, der zugleich als Beitrag der Vereinszeitschrift erschien ${ }^{102}$. Auch für das nachfolgende Kriegsjahr 1914 lässt sich sowohl ein Vortrag Schlegels über Schlafmittel als auch ein schriftlicher Beitrag «zur Kenntnis neuerer Heilmittel» nachweisen ${ }^{103}$.Zudem richtete er am 26. 8. 1914 ein Schreiben an das Medizinalkollegium und an die VereinsLazarette vom Roten Kreuz, in welchem er die Hilfe von Wundärzten in den jeweiligen Lazaretten anbot ${ }^{104}$.

93 Z Wundärzte Geburtsh 59, 1908, S. 178-185.

94 Ebd., S. 213.

95 Z Wundärzte Geburtsh 60, 1909, S. 132 u. 192.

96 Z Wundärzte Geburtsh 61, 1910, S. 3, S. 88, 137, 184, 202, 270.

97 Z Wundärzte Geburtsh 62,1911, S. 177.

98 Ebd., S. 91 u. 210.

99 Z Wundärzte Geburtsh 64, 1913, S. 35.

100 Ebd., S. 93.

101 Wie Anm. 100.

102 Z Wundärzte Geburtsh 64, S. 69, 89, 90, 93.

103 Z Wundärzte Geburtsh 65, 1914, S. 73 u. 101.

104 StAL E 162 I, Bü 459, SSt 145-147. 
Als 1915 mit Gustav Baumgärtel der langjährige Vereinsvorsitzende starb, wurde Arnold Schlegel trotz seines entlegenen Wohnsitzes in der Schweiz für die Nachfolge Baumgärtels vorgeschlagen. Er lehnte jedoch ab und empfahl seinerseits Ernst Bauer aus Renningen als neuen Vorsitzenden ${ }^{105}$. Den Nachruf auf Baumgärtel verfasste Schlegel hingegen selbst; zusätzlich publizierte er 1915 noch sechs weitere Beiträge ${ }^{106}$. Überdies übernahm er 1916 zusammen mit Bauer die Redaktion der Vereinszeitschrift ${ }^{107}$. Im gleichen Jahr war Schlegel mit zwei, 1917 mit einem Artikel vertreten ${ }^{108} .1919$ wurde der <Verein württembergischer Wundärzte und Geburtshelfer> schliesslich wegen Überalterung aufgelöst. Zu jenem Zeitpunkt wurden lediglich noch 18 Wundärzte registriert, wovon 12 dem Verein angehörten ${ }^{109}$. In der letzten Ausgabe der «Zeitschrift für Wundärzte und Geburtshelfer» findet sich unter der Überschrift «Schluss- und Abschiedsworte der Redaktion» ein letzter, 16seitiger Zeitschriftenbeitrag Arnold Schlegels ${ }^{110}$. Daneben enthält besagte Zeitschrift eine Notiz über eine Auszeichnung Schlegels. Elf Jahre nach der ablehnenden Haltung des Medizinalkollegiums wurde Schlegel nunmehr in seiner Eigenschaft als Vorsitzender des «Deutschen Hilfsvereins» in der Schweiz sowie «für seine Tätigkeit in der Fürsorge für Kriegsbeschädigte und Hinterbliebene» die Kgl. preussische Rote-Kreuz-Medaille III. Klasse, das Kgl. Württembergische Wilhelmskreuz sowie das Ritterkreuz des K. Oesterreichischen Franz-Joseph-Ordens verliehen ${ }^{111}$.

Bis zum Ende des Jahres 1921 betrieb Schlegel das Wirtshaus zum Bären weiter. Erst am 18. März 1922 verzichtete er auf die Niederlassungsbewilligung in Einsiedeln und siedelte nach Ravensburg über ${ }^{112}$. Schlegels Aufenthalt in Ravensburg war wiederum sehr kurz; bereits am 23.12. 1922 stellte dieser einen neuerlichen Antrag auf Niederlassung in Einsiedeln. Letztere wurde ihm bewilligt unter der Voraussetzung, dass er «die Steuerpflicht von

105 Z Wundärzte Geburtsh 66, 1915, S. 78.

106 Ebd., S. 33, 57, 69, 74, 77, 76, 84, 87.

107 Seit 1916 tritt Schlegel auf dem Titelblatt der Zeitschrift als Redakteur in Erscheinung.

108 Z Wundärzte Geburtsh 67, 1916, S. 3, 17, 33; 68, 1917, S. 36.

109 Z Wundärzte Geburtsh 70, 1919, S. 12.

110 Ebd., S. 49-64.

111 Ebd., Titelblatt.

112 Protokoll des Bezirksrats Einsiedeln, BI 2. 63, S. 663. Aus dem betreffenden Protokoll ergibt sich, daß Schlegel um Rückzahlung der für das Jahr 1922 bezahlten «Wirtschaftspatenttaxe» ersuchte. Diese hatte er am 30.12.1922 in der Absicht eingelöst, sie zu einem späteren Zeitpunkt auf den neuen Pächter des Bären überschreiben zu lassen. Nachdem Schlegel erfahren habe, dass jener ebenfalls die volle Patenttaxe zu zahlen habe, sei es «nur recht und billig, wenn ihm der Betrag zurückbezahlt werde», da er seit dem 1.1.1922 nicht mehr gewirtet habe. Der Bezirksrat sprach sich gegen eine Rückerstattung aus und vermerkte im Protokoll, Schlegel habe im Gespräch zugestanden, daß er auch 1922 noch «gelegentlich eine Flasche Bier ausgeschenkt habe» (ebenda). 
wenigstens Fr. 15000 in Einsiedeln» anerkenne ${ }^{113}$. Dass Schlegel sich mit jener Bedingung einverstanden erklärte, ergibt sich aus der Tatsache, dass er mit kurzen Unterbrechungen bis zum 1. Juli 1924 in Einsiedeln wohnhaft blieb $^{114}$. Ob er bis zu diesem Zeitpunkt noch einer geregelten Tätigkeit nachging, erscheint zweifelhaft. Das Wirtschaftspatent für das Gasthaus zum Bären war bereits an die Familie Heim-Märky übergegangen ${ }^{15}$.

Nach dem 1. Juli 1924 lebte Schlegel noch für eine kurze Zeit in Ravensburg, wo er am 28.10.1924 verstarb. Interessanterweise enthält die betreffende Akte neben dem Todesdatum den Vermerk, der ständige Wohnsitz Schlegels sei «Einsiedeln/Schweiz» gewesen ${ }^{116}$.

Obgleich Schlegel den grössten Teil seines Lebens als Privatier in der Schweiz verbrachte, galt sein Engagement vor allem der beruflichen Besserstellung der württembergischen Wundärzte. Die Lebensbeschreibung Arnold Schlegels reflektiert dabei in mehrfacher Hinsicht die besondere Situation des aussterbenden Wundarztstandes: So steht Schlegels Tätigkeit als Wirt beispielhaft für viele Wundärzte, die in Württemberg einer fachfremdem (Neben)beschäftigung nachgingen, um so ihre schwierige finanzielle Lage zu verbessern. Sein Engagement als Standespolitiker gibt zudem Aufschluss über die Interessenorganisation und die berufspolitischen Initiativen der württembergischen Wundärzteschaft. Schlegels Petitionen und Monographien belegen darüber hinaus die Unzufriedenheit der Handwerkschirurgen mit der zeitgenössischen Medizinalgesetzgebung, welche von der aussterbenden Berufsgruppe als zunehmende Zurücksetzung und Entrechtung empfunden wurde. Aus den vergeblichen Anträgen Schlegels auf Zulassung zum medizinischen Studium beziehungsweise auf offizielle Anerkennung seines ausländischen Doktortitels spricht ferner der Wunsch nach einer arztähnlichen Stellung und nach einer gesellschaftlichen Aufwertung der wundärztlichen Tätigkeit.

Wie unzufrieden und verbittert Schlegel am Ende über die Entwicklung des Wundarztberufs war, verraten die pathetischen «Schluss- und Abschiedsworte», die er 1919 in der letzten Ausgabe der 〈Zeitschrift für Wundärzte und Geburtshelfer» an die «Stiefkinder Aesculaps» richtete:

«Mehr Ritterklee und Sauerampfer wuchs am Weg, als Süssholz und Tausendguldenkraut, und aus 7 Jahrzehnten blieb manches Vergissmeinnicht.

113 Wie Anm. 111.

114 BAE, Kontrolle der niedergelassenen Ausländer («Zurückzug: 1. Juli 1924»).

115 Vgl. Amtsbl Kanton Schwyz 1922, S. 529.

116 Stadtarchiv Ravensburg, Personal-Karte Arnold Schlegel vom 5. April 1922. 
Fanden wir einmal eine Rose am $\mathrm{Ha}(\mathrm{n}) \mathrm{g}$, so war sie gewiss von der dornigsten Sorte, und wenn wir nun zum Schluss dem freundlichen Leser einen Strauss davon überreichen, dann möge sich niemand daran stechen ${ }^{117}$.»

Mein besonderer Dank gilt Herrn E. Müller, Bezirksverwaltung Einsiedeln, sowie Herrn Lic. B. Zimmermann, Konstanz, für ihre freundliche Unterstïtzung bei der Quellenrecherche.

117 Z Wundärzte Geburtsh 70,1919, S. 50. 